\title{
Eccentric Coloring of a Graph
}

\author{
Medha Itagi Huilgol ${ }^{1} \&$ Syed Asif Ulla S. ${ }^{1}$ \\ ${ }^{1}$ Department of Mathematics, Bangalore University, Central College Campus, Bangalore - 560 001, India. \\ Correspondence: Medha Itagi Huilgol, Department of Mathematics, Bangalore University, Central College Cam- \\ pus, Bangalore - 560 001, India. E-mail: medha@bub.ernet.in
}

Received: October 27, 2014 Accepted: November 14, 2014 Online Published: January 7, 2015

doi:10.5539/jmr.v7n1p1 URL: http://dx.doi.org/10.5539/jmr.v7n1p1

\begin{abstract}
The eccentricity $e(u)$ of a vertex $u$ is the maximum distance of $u$ to any other vertex of $G$. A vertex $v$ is an eccentric vertex of vertex $u$ if the distance from $u$ to $v$ is equal to $e(u)$. An eccentric coloring of a graph $G=(V, E)$ is a function color: $V \rightarrow N$ such that

(i) for all $u, v \in V,(\operatorname{color}(u)=\operatorname{color}(v)) \Rightarrow d(u, v)>\operatorname{color}(u)$.

(ii) for all $v \in V, \operatorname{color}(v) \leq e(v)$.

The eccentric chromatic number $\chi_{e} \in N$ for a graph $G$ is the lowest number of colors for which it is possible to eccentrically color $G$ by colors: $V \rightarrow\left\{1,2, \ldots, \chi_{e}\right\}$. In this paper, we have considered eccentric colorability of a graph in relation to other properties. Also, we have considered the eccentric colorability of lexicographic product of some special class of graphs.
\end{abstract}

Keywords: Eccentricity of a vertex, eccentric vertex, eccentric coloring, eccentric coloring number

2000 Mathematics Subject Classification. Primary 05C12, 05C15, 05 C76.

\section{Introduction}

Unless mentioned otherwise for terminology and notation the reader may refer [Buckley and Harary, 1990] and [Chartrand and Lesniak, 1996], new ones will be introduced as and when found necessary. In this paper we consider simple undirected graphs without multiple edges and self loops. The order $p$ is the number of vertices in $G$ and size $q$ is the number of edges in $G$.

The distance $d(u, v)$ between $u$ and $v$ is the length of a shortest path joining $u$ and $v$. If there exists no path between $u$ and $v$ then we define $d(u, v)=\infty$. The eccentricity $e(u)$ of $u$ is the distance to a vertex farthest from $u$. If $d(u, v)=e(u)(v \neq u)$, we say that $v$ is an eccentric vertex of $u$. The radius $\operatorname{rad}(G)$ is the minimum eccentricity of the vertices, whereas the diameter $\operatorname{diam}(G)$ is the maximum eccentricity. A vertex $v$ is a central vertex if $e(v)=\operatorname{rad}(G)$, and the center $C(G)$ is the set of all central vertices. A graph $G$ is self-centered if $\operatorname{rad}(G)=\operatorname{diam}(G)$.

A chordal graph is a simple graph in which every cycle of length greater than three has a chord. Equivalently, the graph contains no induced cycle of length four or more. The join of two graphs $G_{1}$ and $G_{2}$, defined by Zykov [Zykov, 1949] is denoted $G_{1}+G_{2}$ and consists of $G_{1} \cup G_{2}$ and all edges joining $V_{1}$ with $V_{2}$. For $p \geq 4$, the wheel $W_{p}$ is defined to be the graph $K_{1}+C_{p-1}$. A graph is bipartite if its vertex set can be partitioned into two subsets $X$ and $Y$ so that every edge has one end in $X$ and one end in $Y$; such a partition $(X, Y)$ is called a bipartition of the graph, and $X$ and $Y$ its parts. We denote a bipartite graph $G$ with bipartition $(X, Y)$ by $G[X, Y]$. If $G[X, Y]$ is simple and every vertex in $X$ is joined to every vertex in $Y$, then $G$ is called a complete bipartite graph, denoted by $K_{m, n}$. Clique in a graph is a set of pairwise adjacent vertices. The clique number of a graph $G$, written $\omega(G)$, is the maximum size of a set of pairwise adjacent vertices (clique) in $G$. Many researchers have studied the relations related to clique number [Dirac, 1961], [Fulkerson and Gross, 1965], [Matula, 1972], [Voloshin, 1982], [Voloshin and Gorgos, 1982], etc.

In [Sloper, 2004], Sloper has introduced the concept of eccentric coloring of a graph and studied the eccentric coloring of trees. 
Definition 1.1 (Sloper, 2004). An eccentric coloring of a graph $G=(V, E)$ is a function color: $V \rightarrow N$ such that

(i) for all $u, v \in V,(\operatorname{color}(u)=\operatorname{color}(v)) \Rightarrow d(u, v)>\operatorname{color}(u)$.

(ii) for all $v \in V$, $\operatorname{color}(v) \leq e(v)$.

Definition 1.2 (Sloper, 2004). The eccentric chromatic number $\chi_{e} \in N$ for a graph $G$ is the lowest number of colors for which it is possible to eccentrically color $G$ by colors: $V \rightarrow\left\{1,2, \ldots, \chi_{e}\right\}$.

\section{Bounds on the Radius and Diameter of an Eccentric Colorable Graph}

In this section we determine some bounds on diameter, radius, size of center, clique number, etc. depending on the eccentric colorability of the graph.

Proposition 2.1. In any eccentric colorable graph, there exists at most one vertex with color $c=\operatorname{diam}(G)$.

Proof. Let $G$ be an eccentric colorable graph. Suppose on the contrary, there exist at least two vertices, say, $u$, $v$, with color $c=\operatorname{diam}(G)$. But $d(u, v) \leq \operatorname{diam}(G)$, which contradicts the definition of eccentric colorable graph. Hence, $G$ contains at most one vertex with color $c=\operatorname{diam}(G)$.

In [Negami and XU, 1986], S. Negami et al. have proved that in any 2-self-centered graph, for every vertex $v$ there exists a cycle of length 4 or 5 that preserves the graph distance with a vertex $u$ in that cycle.

Theorem 2.1. If $G$ is a graph with $\operatorname{diam}(G) \leq 2$, then $G$ is not eccentric colorable.

Proof. Let $G$ be a graph with $\operatorname{diam}(G) \leq 2$. Here three cases arise.

Case(i): $\operatorname{rad}(G)=1$ and $\operatorname{diam}(G)=1$.

In this case the graph is a complete graph, which is not eccentric colorable.

Case(ii): $\operatorname{rad}(G)=1$ and $\operatorname{diam}(G)=2$.

Let $u$ be a central vertex of $G$. Since $e(u)=1, u$ can be colored with color 1 only. Let $v$ and $w$ be any two diametral vertices of $G$. The vertices $v$ and $w$ can not be colored with color 1 . Suppose $v$ is colored with color 2 , then $w$ can not be colored with color 2, because $d(v, w)=2$. Hence, $G$ is not eccentric colorable.

Case(iii): $\operatorname{rad}(G)=2$ and $\operatorname{diam}(G)=2$.

In this case the graph $G$ is a self centered graph of radius two. Referring to the paper [Negami and XU, 1986] as cited above, every self centered graph of radius two contains at least four vertices and degree of every vertex is at least two. Let $u$ be any vertex of $G$ and $v, w$ are adjacent to $u$. Since $e(u)=e(v)=e(w)=2$, we can use at most two colors, color 1 and color 2 to color these three vertices. Suppose $u$ is colored with color 1 then any of $v$ and $w$ can not be colored with color 1 and both $v$ and $w$ can not be colored with color 2 . Hence, $G$ is not eccentric colorable. Now suppose $u$ is colored with color 2 then any of $v$ and $w$ can not be colored with color 2. Hence, $v$ and $w$ are colored with color 1 if $v$ and $w$ are not adjacent(If $v$ and $w$ are adjacent then $G$ is not eccentric colorable). Since $\operatorname{deg}(v) \geq 2$, there exists a vertex say, $x$ adjacent to $v$. The vertex $x$ can not be colored with color 1 or color 2 , since $d(u, x) \leq 2$ and $d(v, x) \leq 1$. Hence, $G$ is not eccentric colorable.

Note: The converse of Theorem 2.1 need not be true.

For example, $C_{7}\left[\bar{K}_{2}\right]$ is of diameter 3 , which is not eccentric colorable.

Remark 1. The following graphs are not eccentric colorable,

(i) Connected, self centered, chordal graph,

(ii) Petersen graph,

(iii) Graph with radius one,

For example, $W_{1, p-1}, p-1 \geq 1$.

(iv) Complete bipartite graph $K_{m, n}, m, n \geq 1$.

Lemma 2.1. If a graph $G$ containing the wheel $W_{1, p-1}$ is eccentric colorable then $\chi_{e}(G) \geq 2+\left\lceil\frac{p-1}{2}\right\rceil$.

Proof. Let a graph $G$ containing the wheel $W_{1, p-1}$ be eccentric colorable. The subgraph $W_{1, p-1}$ requires at least $2+\left\lceil\frac{p-1}{2}\right\rceil$ colors as shown in Figure 1. Hence, $\chi_{e}(G) \geq 2+\left\lceil\frac{p-1}{2}\right\rceil$. 


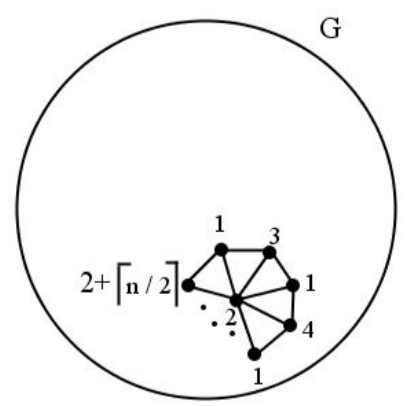

Figure 1. Graph $G$ containing a wheel

Proposition 2.2. If a graph $G$ is eccentric colorable then $|C(G)| \leq \operatorname{rad}(G)$.

Proof. Let a graph $G$ be eccentric colorable. Suppose on the contrary $|C(G)|>\operatorname{rad}(G)$, then the vertices of $C(G)$ require at least $\operatorname{rad}(G)+1$ distinct colors but the eccentricity of every central vertex is $\operatorname{rad}(G)$. Hence, $G$ is not eccentric colorable, a contradiction. Hence, $|C(G)| \leq \operatorname{rad}(G)$.

Proposition 2.3. If a graph $G$ is eccentric colorable then $\omega(G) \leq \operatorname{diam}(G)$, where $\omega(G)$ is the clique number of $G$.

Proof. Let a graph $G$ be eccentric colorable. Suppose on the contrary $\omega(G)>\operatorname{diam}(G)$ then the vertices of maximum clique require at least $\operatorname{diam}(G)+1$ distinct colors, but eccentricity of every vertex of $G$ is at most $\operatorname{diam}(G)$. Hence, $G$ is not eccentric colorable, a contradiction. Hence, $\omega(G) \leq \operatorname{diam}(G)$.

Theorem 2.2. If a graph $G$ is eccentric colorable then $G$ contains no $K_{m, n}, m, n \geq \operatorname{diam}(G)$ as its subgraph.

Proof. Let a graph $G$ be eccentric colorable. Suppose on the contrary $G$ contains $K_{m, n}$, where $m, n \geq \operatorname{diam}(G)$ as subgraph then the vertices of $K_{m, n}$ require at least $\operatorname{diam}(G)+1$ colors, but $e(v) \leq \operatorname{diam}(G)$ for all $v \in V(G)$, a contradiction to the fact that $G$ is eccentric colorable. Hence, $G$ contains no $K_{m, n}, m, n \geq \operatorname{diam}(G)$ as its subgraph.

Theorem 2.3. There exists no connected graph $G$ with $\chi_{e}(G)=2$.

Proof. For $\chi_{e}(G)=2, G$ must contain at least two vertices. The only connected graph on two vertices is $K_{2}$, which is not eccentric colorable and every connected graph with at least three vertices contains a path $P_{3}$ which requires at least three colors. Hence, there exists no connected graph $G$ with $\chi_{e}(G)=2$.

Remark 2. Any disconnected graph is eccentric colorable, since the eccentricity of every vertex in $G$ is infinite.

\section{Eccentric Coloring of Cycle With Chord}

In this section we obtain results on eccentric colorability of a cycle with a chord between two vertices at distance two and eccentric colorability of a cycle with a chord between two vertices at distance three.

Theorem 3.1. For $p \geq 8$,

$$
\chi_{e}\left(C_{p}\right)= \begin{cases}3, & \text { if } p=4(n+1), n \geq 1 \\ 4, & \text { otherwise. }\end{cases}
$$

Proof. If $p=4(n+1), n \geq 1$, color the vertices of $C_{p}$ with the sequence $1213, \ldots$ till all the vertices are colored. The resulting coloring is eccentric coloring with $\chi_{e}\left(C_{p}\right)=3$, since for any connected graph $\chi_{e}(G) \geq 3$.

If $p \neq 4(n+1), n \geq 1$, color the vertices of $C_{p}$ with the sequence $1213, \ldots$ till $p-1$ vertex and color the vertex $v_{p}$ with color 4 . The resulting coloring is eccentric coloring with $\chi_{e}\left(C_{p}\right)=4$,

Lemma 3.1. A cycle $C_{p}, p \geq 9$ with a chord between two vertices at distance two from each other is eccentric colorable. 
Proof. Let a cycle $C_{p}, p \geq 9$ be labeled as $v_{1}, v_{2}, v_{3}, \ldots, v_{p}$. Let $e$ be a chord between $v_{p}$ and $v_{2}$. Eccentric coloring is given as follows:

Case(i): $p=4 m+5, m \geq 1$.

In this case, the eccentric coloring is given by

The vertices in the sets

$A=\left\{v_{2 n-1} / 1 \leq n \leq \frac{p-1}{2}\right\}$,

$B=\left\{v_{4 n-2} / 1 \leq n \leq \frac{p-1}{4}\right\}$,

$C=\left\{v_{4 n} / 1 \leq n \leq \frac{p-5}{4}\right\} \cup\left\{v_{p}\right\}$, and

$D=\left\{v_{p-1}\right\}$ are colored with color $1,3,2$, and 4, respectively.

Case(ii): $p=4 m+6, m \geq 1$.

In this case, the eccentric coloring is given by

The vertices in the sets

$A=\left\{v_{2 n-1} / 1 \leq n \leq \frac{p}{2}\right\}$,

$B=\left\{v_{4 n-2} / 1 \leq n \leq \frac{p-2}{4}\right\}$,

$C=\left\{v_{4 n} / 1 \leq n \leq \frac{p-6}{4}\right\} \cup\left\{v_{p}\right\}$, and

$D=\left\{v_{p-2}\right\}$ are colored with color $1,3,2$, and 4 , respectively.

Case(iii): $p=4 m+7, m \geq 1$.

In this case, the eccentric coloring is given by

The vertices in the sets

$A=\left\{v_{2 n-1} / 1 \leq n \leq \frac{p-1}{2}\right\}$,

$B=\left\{v_{4 n-2} / 1 \leq n \leq \frac{p-3}{4}\right\}$,

$C=\left\{v_{4 n} / 1 \leq n \leq \frac{p-3}{4}\right\} \cup\left\{v_{p}\right\}$, and

$D=\left\{v_{p-1}\right\}$ are colored with color $1,3,2$, and 4 , respectively.

Case(iv): $p=4 m+8, m \geq 1$.

In this case, the eccentric coloring is given by

The vertices in the sets

$A=\left\{v_{2 n-1} / 1 \leq n \leq \frac{p}{2}\right\}$,

$B=\left\{v_{4 n-2} / 1 \leq n \leq \frac{p-4}{4}\right\}$,

$C=\left\{v_{4 n} / 1 \leq n \leq \frac{p-4}{4}\right\} \cup\left\{v_{p}\right\}$, and

$D=\left\{v_{p-2}\right\}$ are colored with color $1,3,2$, and 4, respectively.

For example, let us consider the graphs as shown in Figure 2.

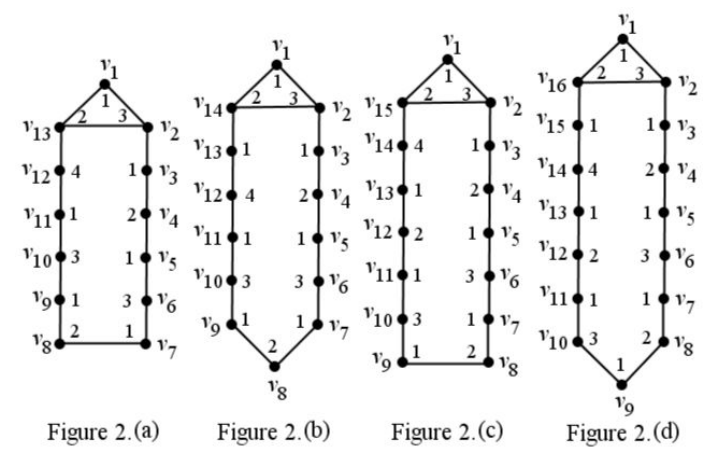

Figure 2. Cycle with chord between vertices at distance two

Throughout this example we consider $m=2$.

For $p=4 m+5=13$, the graph is shown in Figure 2(a) and its eccentric coloring is as follows:

The vertices in the sets

$A=\left\{v_{2 n-1} / 1 \leq n \leq \frac{p-1}{2}\right\}=\left\{v_{1}, v_{3}, v_{5}, v_{7}, v_{9}, v_{11}\right\}$,

$B=\left\{v_{4 n-2} / 1 \leq n \leq \frac{p-1}{4}\right\}=\left\{v_{2}, v_{6}, v_{10}\right\}$,

$C=\left\{v_{4 n} / 1 \leq n \leq \frac{p-5}{4}\right\} \cup\left\{v_{p}\right\}=\left\{v_{4}, v_{8}\right\} \cup\left\{v_{13}\right\}$, and 
$D=\left\{v_{p-1}\right\}=\left\{v_{12}\right\}$ are colored with colors $1,3,2$, and 4, respectively.

Similar cases are shown with their respective colors, in Figure 2(b), 2(c), 2(d) respectively.

Lemma 3.2. A cycle $C_{p}, p \geq 9$ with a chord between two vertices at distance three from each other is eccentric colorable.

Proof. Let a cycle $C_{p}, p \geq 9$ be labeled as $v_{1}, v_{2}, v_{3}, \ldots, v_{p}$. Let $e$ be a chord between $v_{p-1}$ and $v_{2}$. Eccentric coloring is given as follows:

Case(i): $p=4 m+5, m \geq 1$.

In this case, the eccentric coloring is given by

The vertices in the sets

$A=\left\{v_{2 n-1} / 1 \leq n \leq \frac{p-1}{2}\right\}$,

$B=\left\{v_{4 n-2} / 1 \leq n \leq \frac{p-1}{4}\right\}$,

$C=\left\{v_{4 n} / 1 \leq n \leq \frac{p-5}{4}\right\} \cup\left\{v_{p}\right\}$, and

$D=\left\{v_{p-1}\right\}$ are colored with colors $1,2,3$, and 4 , respectively.

Case(ii): $p=4 m+6, m \geq 1$.

In this case, the eccentric coloring is given by

The vertices in the sets

$A=\left\{v_{2 n-1} / 1 \leq n \leq \frac{p}{2}\right\}$,

$B=\left\{v_{4 n-2} / 1 \leq n \leq \frac{p-2}{4}\right\}$,

$C=\left\{v_{4 n} / 1 \leq n \leq \frac{p-2}{4}\right\}$, and

$D=\left\{v_{p}\right\}$ are colored with colors $1,2,3$, and 4 , respectively.

Case(iii): $p=4 m+7, m \geq 1$.

In this case, the eccentric coloring is given by

The vertices in the sets

$A=\left\{v_{2 n-1} / 1 \leq n \leq \frac{p-1}{2}\right\}$,

$B=\left\{v_{4 n-2} / 1 \leq n \leq \frac{p-3}{4}\right\}$,

$C=\left\{v_{4 n} / 1 \leq n \leq \frac{p-3}{4}\right\}$,

$D=\left\{v_{p-1}\right\}$, and

$E=\left\{v_{p}\right\}$ are colored with colors $1,2,3,4$, and 5, respectively.

Case(iv): $p=4 m+8, m \geq 1$.

In this case, the eccentric coloring is given by

The vertices in the sets

$A=\left\{v_{2 n-1} / 1 \leq n \leq \frac{p}{2}\right\}$,

$B=\left\{v_{4 n-2} / 1 \leq n \leq \frac{p-4}{4}\right\}$,

$C=\left\{v_{4 n} / 1 \leq n \leq \frac{p}{4}\right\}$

$D=\left\{v_{p-2}\right\}$ are colored with colors $1,2,3$, and 4 , respectively.

For example, let us consider the graphs as shown in Figure 3. In this example too we consider $m=2$.

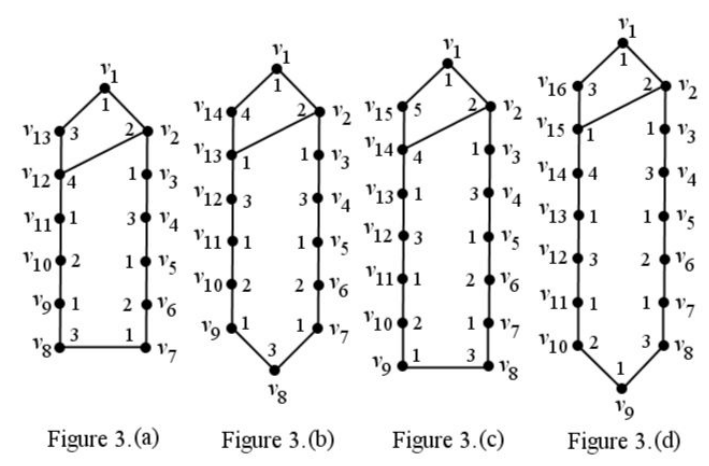

Figure 3. Cycle with chord between vertices at distance three 
For $p=4 m+5=13$, the graph is shown in Figure 3(a) and its eccentric coloring is as follows:

The vertices in the sets

$A=\left\{v_{2 n-1} / 1 \leq n \leq \frac{p-1}{2}\right\}=\left\{v_{1}, v_{3}, v_{5}, v_{7}, v_{9}, v_{11}\right\}$,

$B=\left\{v_{4 n-2} / 1 \leq n \leq \frac{p-1}{4}\right\}=\left\{v_{2}, v_{6}, v_{10}\right\}$,

$C=\left\{v_{4 n} / 1 \leq n \leq \frac{p-5}{4}\right\} \cup\left\{v_{p}\right\}=\left\{v_{4}, v_{8}\right\} \cup\left\{v_{13}\right\}$, and

$D=\left\{v_{p-1}\right\}=\left\{v_{12}\right\}$ are colored with colors $1,2,3$, and 4 , respectively.

Similarly, the other cases are shown with their colorings in Figure 3(b), 3(c), 3(d) respectively.

\section{Eccentric Coloring of Lexicographic Products}

In this section we consider the eccentric coloring of lexicographic product of class of graphs. First, we define lexicographic product as follows:

Given graphs $G$ and $H$, the lexicographic product $G[H]$ has vertex set $\{(g, h): g \in V(G), h \in V(H)\}$ and two vertices $(g, h),\left(g^{\prime}, h^{\prime}\right)$ are adjacent if and only if either $\left[g, g^{\prime}\right]$ is an edge of $G$ or $g=g^{\prime}$ and $\left[h, h^{\prime}\right]$ is an edge of $H$.

Theorem 4.1. For any even integer $p \geq 16, C_{p}\left[\bar{K}_{2}\right]$ is eccentric colorable.

Proof. Let a cycle $C_{p}$ be labeled as $v_{1}, v_{2}, v_{3}, \ldots, v_{p}$, where $p(\geq 16)$ is an even integer and $C_{p}\left[\bar{K}_{2}\right]$ be the lexicographic product of $C_{p}$ and $\bar{K}_{2}$. Let $S_{i}$ denote the set of vertices of $\bar{K}_{2}$ replaced in the place of $v_{i}$.

Case(i): $p=8 n+8, n \geq 1$.

In this case, let $V\left(C_{p}\left[\bar{K}_{2}\right]\right)=A \cup B \cup C \cup D$ be the partition of $V\left(C_{p}\left[\bar{K}_{2}\right]\right)$, where

$A=\left\{\cup S_{2 k-1} / 1 \leq k \leq \frac{p}{2}\right\}$,

$B=\left\{\cup S_{4 k-2} / 1 \leq k \leq \frac{p}{4}\right\}$,

$C=\left\{\cup S_{8 k-2} / 1 \leq k \leq \frac{p}{8}\right\}$,

$D=\left\{\cup S_{8 k} / 1 \leq k \leq \frac{p}{8}\right\}$.

The eccentric coloring of $C_{p}\left[\bar{K}_{2}\right]$ is given as follows:

The pair of vertices in each $S_{2 k-1}, 1 \leq k \leq \frac{p}{2}$ in $A$ are colored with color 1. Among the pair of vertices in each $S_{4 k-2}, 1 \leq k \leq \frac{p}{4}$ in $B$, one vertex is colored with color 1 and the other vertex with color 2 . Among the pair of vertices in each $S_{4 k-4}, 1 \leq k \leq \frac{p}{8}$ in $C$, one vertex is colored with color 4 and the other vertex with color 5 . Among the pair of vertices in each $S_{8 k}, 1 \leq k \leq \frac{p}{8}$ in $D$, one vertex is colored with color 6 and the other vertex with color 7.

Case(ii): $p=8 n+10, n \geq 1$.

In this case, let $V\left(C_{p}\left[\bar{K}_{2}\right]\right)=A \cup B \cup C \cup D \cup E$ be the partition of $V\left(C_{p}\left[\bar{K}_{2}\right]\right)$, where

$A=\left\{\cup S_{2 k-1} / 1 \leq k \leq \frac{p}{2}\right\}$,

$B=\left\{\cup S_{4 k-2} / 1 \leq k \leq \frac{p-2}{4}\right\}$,

$C=\left\{\cup S_{8 k-4} / 1 \leq k \leq \frac{p-2}{8}\right\}$,

$D=\left\{\cup S_{8 k} / 1 \leq k \leq \frac{p-2}{8}\right\}$, and

$E=\left\{S_{p}\right\}$.

The eccentric coloring of $C_{p}\left[\bar{K}_{2}\right]$ is given as follows:

The pair of vertices in each $S_{2 k-1}, 1 \leq k \leq \frac{p}{2}$, are colored with color 1 .

Among the pair of vertices in each $S_{4 k-2}, 1 \leq k \leq \frac{p-2}{4}$ in $B$, one vertex is colored with color 2 and the other vertex with color 3. Among the pair of vertices in each $S_{8 k-4}, 1 \leq k \leq \frac{p-2}{8}$ in $C$, one vertex is colored with color 4 and the other vertex with color 5 . Among the pair of vertices in each $S_{8 k}, 1 \leq k \leq \frac{p-2}{8}$ in $D$, one vertex is colored with color 6 and the other vertex with color 7. Among the pair of vertices in $S_{p}$ in $E$, one vertex is colored with color 8 and the other vertex with color 9 .

Case(iii): $p=8 n+12, n \geq 1$.

In this case, let $V\left(C_{p}\left[\bar{K}_{2}\right]\right)=A \cup B \cup C \cup D \cup E$ be the partition of $V\left(C_{p}\left[\bar{K}_{2}\right]\right)$, where

$A=\left\{\cup S_{2 k-1} / 1 \leq k \leq \frac{p}{2}\right\}$,

$B=\left\{\cup S_{4 k-2} / 1 \leq k \leq \frac{p}{4}\right\}$,

$C=\left\{\cup S_{8 k-4} / 1 \leq k \leq \frac{p-4}{8}\right\}$,

$D=\left\{\cup S_{8 k} / 1 \leq k \leq \frac{p-4}{8}\right\}$,

$E=\left\{S_{p}\right\}$.

The eccentric coloring of $C_{p}\left[\bar{K}_{2}\right]$ is given as follows: 
The pair of vertices in each $S_{2 k-1}, 1 \leq k \leq \frac{p}{2}$, are colored with color 1 .

Among the pair of vertices in each $S_{4 k-2}, 1 \leq k \leq \frac{p}{4}$ in $B$, one vertex is colored with color 2 and the other vertex with color 3. Among the pair of vertices in each $S_{8 k-4}, 1 \leq k \leq \frac{p-4}{8}$ in $C$, one vertex is colored with color 4 and the other vertex with color 5 . Among the pair of vertices in each $S_{8 k}, 1 \leq k \leq \frac{p-4}{8}$ in $D$, one vertex is colored with color 6 and the other vertex with color 7. Among the pair of vertices in $S_{p}$ in $E$, one vertex is colored with color 8 and the other vertex with color 9 .

Case(iv): $p=8 n+14, n \geq 1$.

In this case, let $V\left(C_{p}\left[\bar{K}_{2}\right]\right)=A \cup B \cup C \cup D \cup E$ be the partition of $V\left(C_{p}\left[\bar{K}_{2}\right]\right)$, where

$A=\left\{\cup S_{2 k-1} / 1 \leq k \leq \frac{p}{2}\right\}$,

$B=\left\{\cup S_{4 k-2} / 1 \leq k \leq \frac{p-2}{4}\right\}$,

$C=\left\{\cup S_{8 k-4} / 1 \leq k \leq \frac{p+2}{8}\right\}$,

$D=\left\{\cup S_{8 k} / 1 \leq k \leq \frac{p-6}{8}\right\}$,

$E=\left\{S_{p}\right\}$.

The eccentric coloring of $C_{p}\left[\bar{K}_{2}\right]$ is given as follows:

The pair of vertices in each $S_{2 k-1}, 1 \leq k \leq \frac{p}{2}$, are colored with color 1 .

Among the pair of vertices in each $S_{4 k-2}, 1 \leq k \leq \frac{p-2}{4}$ in $B$, one vertex is colored with color 2 and the other vertex with color 3. Among the pair of vertices in each $S_{8 k-4}, 1 \leq k \leq \frac{p+2}{8}$ in $C$, one vertex is colored with color 4 and the other vertex with color 5. Among the pair of vertices in each $S_{8 k}, 1 \leq k \leq \frac{p-6}{8}$ in $D$, one vertex is colored with color 6 and the other vertex with color 7. Among the pair of vertices in $S_{p}$ in $E$, one vertex is colored with color 8 and the other vertex with color 9 .

For example, let us consider $C_{16}\left[\bar{K}_{2}\right], C_{18}\left[\bar{K}_{2}\right], C_{20}\left[\bar{K}_{2}\right]$ and $C_{22}\left[\bar{K}_{2}\right]$ as shown in Figure 4 and Figure 5.

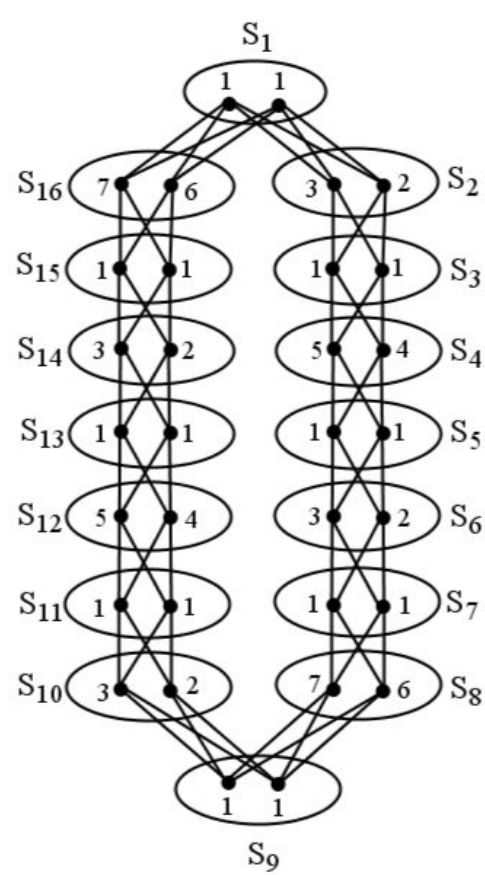

Figure 4.(a)

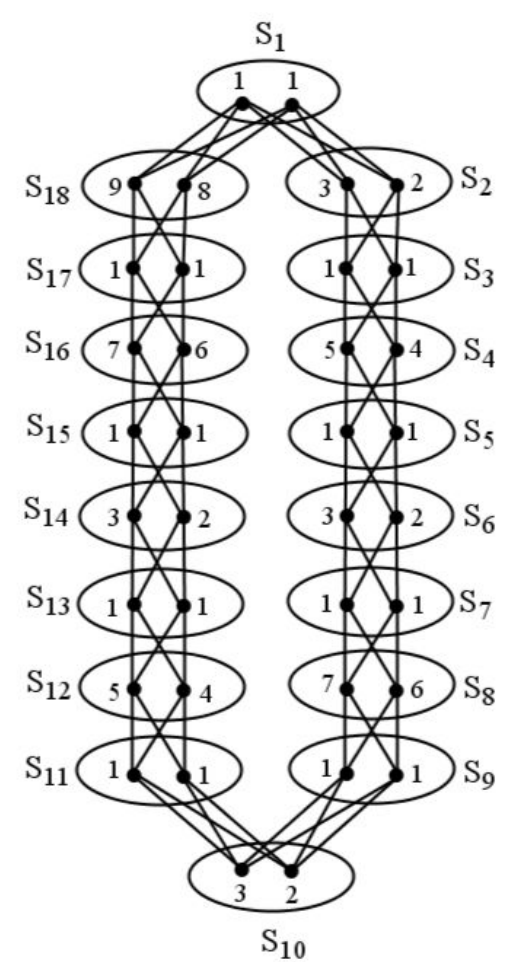

Figure 4.(b)

Figure 4. Lexicographic product $C_{16}\left[\bar{K}_{2}\right]$ and $C_{18}\left[\bar{K}_{2}\right]$

For $p=8 n+8=16, V\left(C_{16}\left[\bar{K}_{2}\right]\right)=A \cup B \cup C \cup D$, where

$A=\left\{\cup S_{2 k-1} / 1 \leq k \leq \frac{p}{2}\right\}=\left\{S_{1}, S_{3}, S_{5}, S_{7}, S_{9}, S_{11}, S_{13}, S_{15}\right\}$,

$B=\left\{\cup S_{4 k-2} / 1 \leq k \leq \frac{p}{4}\right\}=\left\{S_{2}, S_{6}, S_{10}, S_{14}\right\}$, 
$C=\left\{\cup S_{8 k-4} / 1 \leq k \leq \frac{p}{8}\right\}=\left\{S_{4}, S_{12}\right\}$,

$D=\left\{\cup S_{8 k} / 1 \leq k \leq \frac{p}{8}\right\}=\left\{S_{8}, S_{16}\right\}$.

Using Theorem 4.1, the eccentric coloring of $C_{16}\left[\bar{K}_{2}\right]$ is shown in Figure 4(a).

For $p=8 n+10=18$, let $V\left(C_{18}\left[\bar{K}_{2}\right]\right)=A \cup B \cup C \cup D \cup E$, where

$A=\left\{\cup S_{2 k-1} / 1 \leq k \leq \frac{p}{2}\right\}=\left\{S_{1}, S_{3}, S_{5}, S_{7}, S_{9}, S_{11}, S_{13}, S_{15}, S_{17}\right\}$,

$B=\left\{\cup S_{4 k-2} / 1 \leq k \leq \frac{p-2}{4}\right\}=\left\{S_{2}, S_{6}, S_{10}, S_{14}\right\}$,

$C=\left\{\cup S_{8 k-4} / 1 \leq k \leq \frac{p-2}{8}\right\}=\left\{S_{4}, S_{12}\right\}$,

$D=\left\{\cup S_{8 k} / 1 \leq k \leq \frac{p-2}{8}\right\}=\left\{S_{8}, S_{16}\right\}$, and

$E=\left\{S_{p}\right\}=\left\{S_{18}\right\}$.

Using Theorem 4.1, the eccentric coloring of $C_{18}\left[\bar{K}_{2}\right]$ is shown in Figure 4(b).

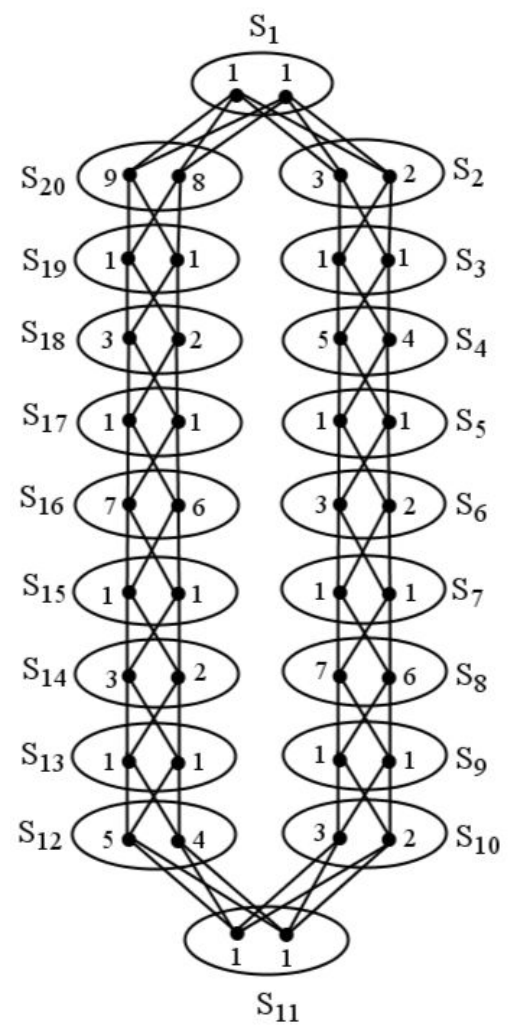

Figure 5.(a)

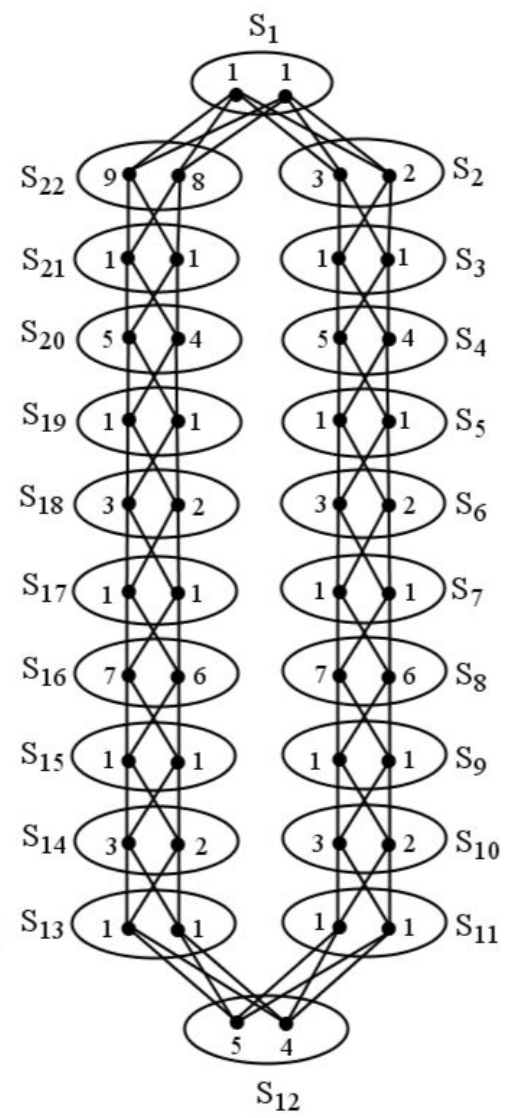

Figure 5.(b)

Figure 5. Lexicographic product $C_{20}\left[\bar{K}_{2}\right]$ and $C_{22}\left[\bar{K}_{2}\right]$

For $p=8 n+12=20$, let $V\left(C_{p}\left[\bar{K}_{2}\right]\right)=A \cup B \cup C \cup D \cup E$, where

$A=\left\{\cup S_{2 k-1} / 1 \leq k \leq \frac{p}{2}\right\}=\left\{S_{1}, S_{3}, S_{5}, S_{7}, S_{9}, S_{11}, S_{13}, S_{15}, S_{17}, S_{19}\right\}$,

$B=\left\{\cup S_{4 k-2} / 1 \leq k \leq \frac{p}{4}\right\}=\left\{S_{2}, S_{6}, S_{10}, S_{14}, S_{18}\right\}$,

$C=\left\{\cup S_{8 k-4} / 1 \leq k \leq \frac{p-4}{8}\right\}=\left\{S_{4}, S_{12}\right\}$,

$D=\left\{\cup S_{8 k} / 1 \leq k \leq \frac{p-4}{8}\right\}=\left\{S_{8}, S_{16}\right\}$,

$E=\left\{S_{p}\right\}=\left\{S_{20}\right\}$.

Using Theorem 4.1, the eccentric coloring of $C_{20}\left[\bar{K}_{2}\right]$ is shown in Figure 5(a).

For $p=8 n+14=22$, let $V\left(C_{p}\left[\bar{K}_{2}\right]\right)=A \cup B \cup C \cup D \cup E$, where

$A=\left\{\cup S_{2 k-1} / 1 \leq k \leq \frac{p}{2}\right\}=\left\{S_{1}, S_{3}, S_{5}, S_{7}, S_{9}, S_{11}, S_{13}, S_{15}, S_{17}, S_{19}, S_{21}\right\}$,

$B=\left\{\cup S_{4 k-2} / 1 \leq k \leq \frac{p-2}{4}\right\}=\left\{S_{2}, S_{6}, S_{10}, S_{14}, S_{18}\right\}$,

$C=\left\{\cup S_{8 k-4} / 1 \leq k \leq \frac{p+2}{8}\right\}=\left\{S_{4}, S_{12}, S_{20}\right\}$,

$D=\left\{\cup S_{8 k} / 1 \leq k \leq \frac{p-6}{8}\right\}=\left\{S_{8}, S_{16}\right\}$, 
$E=\left\{S_{p}\right\}=\left\{S_{22}\right\}$.

Using Theorem 4.1, the eccentric coloring of $C_{22}\left[\bar{K}_{2}\right]$ is shown in Figure 5(b).

\section{References}

Bouchemakh, I., \& Zemir, M. (2012). On the Broadcast Independence Number of Grid Graph. Graphs and Combinatorics, 8.

Buckley, F., \& Harary, F. (1990). Distance in Graphs. Addison Wesley.

Chartrand, G., \& Lesniak, L. (1996). Graphs and Digraphs, 3rd Ed., Chapman \& Hall, London.

Dirac, G. A. (1961). On rigid circuit graphs. Abh. Math. Sem. Univ., Hamburg, 25, 71-76. http://dx.doi.org/10.1007/BF02992776

Dunbar, J. E., Erwin, D. J., Haynes, T. W., Hedetniemi, S. M., \& Hedetniemi, S. T. (2006). Broadcasts in graphs. Discrete Applied Mathematics, 154, 59-75. http://dx.doi.org/10.1016/j.dam.2005.07.009

Fulkerson, D. R., \& Gross, O. A. (1965). Incidence matrices and interval graphs. Pac. J. Math., 15, 835-855. http://dx.doi.org/10.2140/pjm.1965.15.835

Matula, D. W. (1972). The employee party problem. Not. A.M.S., 19, A-382.

Negami, S., \& XU, G. H. (1986). Locally geodesic cycles in 2-self-centered graphs. Discrete Mathematics, 58, 263-268. http://dx.doi.org/10.1016/0012-365X(86)90143-3

Nieminen, J. (1989). The center problem in the product of graphs. Recent Studies in Graph Theory, Vishwa International Publication, 201-205.

Sabidussi, G. (1961). Graph derivatives, Math.Z., 76, 385-401. http://dx.doi.org/10.1007/BF01210984

Sloper, C. (2004). An eccentric coloring of trees. Australasian Journal of Combinatorics, 29, 309-321.

Voloshin, V. I. (1982). Properties of triangulated graphs(Russian). Operations research and programming. (ed. B. A. Shcherbakov) Shtiintsa, 24-32.

Voloshin, V. I., \& Gorgos, I. M. (1982). Some properties of 1-simply connected hypergraphs and their applications (Russian), in Graphs, hypergraphs and discrete optimization problems. Mat. Issled., 66, 30-33.

Zykov, A. A. (1949). On some properties of linear complexes. (Russian) Mat. Sbornik, 24, 163-188.

\section{Copyrights}

Copyright for this article is retained by the author(s), with first publication rights granted to the journal.

This is an open-access article distributed under the terms and conditions of the Creative Commons Attribution license (http://creativecommons.org/licenses/by/3.0/). 